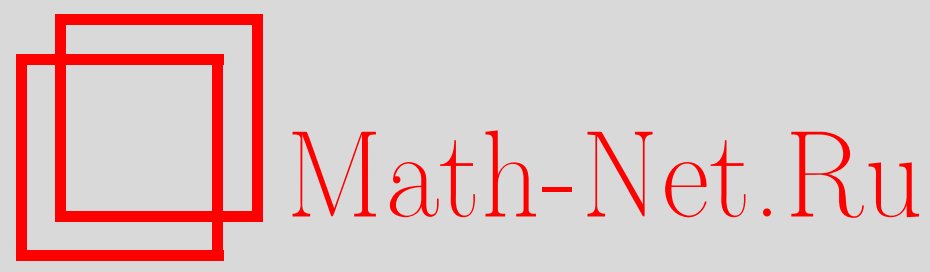

И. А. Кравченко, О числе совпадений двух однородных случайных блужданий с положительными приращениями, Дискрет. матем., 2010, том 22, выпуск 3, 63-74

DOI: https://doi.org/10.4213/dm1107

Использование Общероссийского математического портала Math-Net.Ru подразумевает, что вы прочитали и согласны с пользовательским соглашением http://www . mathnet.ru/rus/agreement

Параметры загрузки:

IP : 54.164 .48 .24

26 апреля 2023 г., 14:01:40 


\title{
О числе совпадений двух однородных случайных блужданий с положительными приращениями
}

\author{
() 2010 г. И. А. Кравченко
}

\begin{abstract}
В работе изучается распределение случайной величины, равной числу совпадений двух однородных случайных блужданий с положительными независимыми приращениями. Данная случайная величина есть длина подпоследовательности общих элементов в двух случайных последовательностях, являющихся случайными подпоследовательностями одной и той же случайной последовательности. Для рассматриваемой случайной величины получено асимптотическое выражение для ее математического ожидания, а также установлена предельная теорема в предположении существования конечной дисперсии у последовательных промежутков между совпадениями двух случайных блужданий. Для частного случая случайных блужданий с приращениями 1 и 2 доказана конечность указанной дисперсии и получено ее выражение через параметры распределения случайных блужданий.
\end{abstract}

\section{1. Введение}

Исследованиям вероятностных свойств последовательных совпадений знаков в различных случайных последовательностях посвящено ряд работ. В работе [1] получены оценки для математического ожидания максимальной длины общей подпоследовательности двух независимых последовательностей равновероятных испытаний. В работах $[2,3]$ в контексте решения криптографических задач, упомянутых в [4], изучалось совместное распределение числа плотных серий в случайной последовательности со значениями в конечном алфавите, в частности, получены предельные теоремы для числа плотных серий единиц заданных длин и длины, не меньшей заданной, а также для максимальной длины плотной серии единиц.

Одна из задач, возникающих при наблюдении в случайные моменты времени множества объектов, характеризующихся соответствующими последовательностями своих состояний, состоит в исследовании распределения длины подпоследовательности общих элементов двух случайных последовательностей, которые, в свою очередь, являются случайными подпоследовательностями одной и той же случайной последовательности.

Рассмотрим два случайных блуждания на множестве натуральных чисел:

$$
\tau_{i}=\left\{\tau_{i}(t)\right\}_{t=1}^{\infty}, \quad i=1,2
$$


Здесь

$$
\tau_{i}(t)=\sum_{r=1}^{t} h_{r}^{(i)}, \quad t=1,2, \ldots,
$$

где $\left\{h_{r}^{(i)}\right\}_{r=1}^{\infty}-$ независимые в совокупности одинаково распределенные случайные величины; распределение $h_{1}^{(i)}$ невырождено и сосредоточено на решетке натуральных чисел с максимальным шагом 1 ; математическое ожидание $a$ случайной величины $h_{1}^{(i)}$ конечно, $a>1 ; i=1,2 ; \tau_{1}, \tau_{2}$ независимы, и распределения $h_{1}^{(1)}, h_{1}^{(2)}$ совпадают.

Определим случайную величину

$$
L_{N, M}=\sum_{t=1}^{N} \sum_{m=1}^{M} I\left(\tau_{1}(t)=\tau_{2}(m)\right),
$$

где $I\left(\tau_{1}(t)=\tau_{2}(m)\right)$ - индикатор события $\left\{\tau_{1}(t)=\tau_{2}(m)\right\} ; t \geqslant 1 ; m \geqslant 1$. Очевидно, $L_{N, M}$ есть число совпадений двух однородных случайных блужданий $\tau_{1}, \tau_{2}$ с положительными приращениями на отрезках натуральных чисел $\{1, \ldots, N\},\{1, \ldots, M\}$ соответственно.

\section{2. Основные результаты}

Относительно математического ожидания $L_{N, M}$ верна следующая теорема.

Теорема 1. Пусть $\mathbf{E}\left(h_{1}^{(1)}\right)^{4}<\infty$. Тогда, если $N, M \rightarrow \infty$ так, что величина $(N-M) /(N+M)^{1 / 2}$ сходится к конечному числу $\lambda$, то имеет место асимптотическое равенство

$$
\mathbf{E} L_{N, M}=\frac{N+M}{2 a}\left(1+O\left(\frac{\ln (N+M)}{(N+M)^{1 / 2}}\right)\right) .
$$

Доказательство. Получим выражение для математического ожидания случайной величины $L_{N, M}$, считая, без ограничения общности, что $M \geqslant N$.

$$
\begin{aligned}
\mathbf{E} L_{N, M} & =\sum_{t=1}^{N} \sum_{m=1}^{M} P(t, m) \\
& =\sum_{n=2}^{N} \sum_{t=1}^{n-1} P(t, n-t)+\sum_{n=N+1}^{M} \sum_{t=1}^{N} P(t, n-t)+\sum_{n=M+1}^{N+M} \sum_{t=n-M}^{N} P(t, n-t),
\end{aligned}
$$

где

$$
P(t, m)=\mathbf{P}\left(\tau_{1}(t)=\tau_{2}(m)\right)
$$

- вероятность того, что случайные последовательности $\tau_{1}$ и $\tau_{2}$ совпадают в моменты $t$ и $m$ соответственно, $t \geqslant 1, m \geqslant 1$.

Введем обозначение

$$
Q_{n}(k, r)=\sum_{t=k}^{r} P(t, n-t),
$$

где $k \geqslant 1, r \geqslant 1, n \geqslant k+1, n \geqslant r+1$, при этом $Q_{n}(k, r)=0$, если $k<r$. 
Тогда

$$
\mathbf{E} L_{N, M}=\sum_{n=2}^{N} Q_{n}(1, n-1)+\sum_{n=N+1}^{M} Q_{n}(1, N)+\sum_{n=M+1}^{N+M} Q_{n}(n-M, N) .
$$

Вероятность $P(t, n-t)$ есть вероятность того, что сумма $n$ независимых решетчатых случайных величин $\left\{H_{1}^{(1)}, \ldots, H_{t}^{(1)}, H_{1}^{(2)}, \ldots, H_{n-t}^{(2)}\right\}$ равна 0 . При этом распределение $H_{1}^{(1)}, \ldots, H_{t}^{(1)}$ одно и то же и совпадает с распределением $h_{1}^{(1)}$. Распределение $H_{1}^{(2)}, \ldots, H_{n-t}^{(2)}$ одно и то же и совпадает с распределением $-h_{1}^{(1)}$. Распределения случайных величин $\left\{H_{1}^{(1)}, \ldots, H_{t}^{(1)}, H_{1}^{(2)}, \ldots, H_{n-t}^{(2)}\right\}$ сосредоточены на решетке целых чисел с максимальным шагом, равным 1 . В силу условия теоремы $\mathbf{E}\left(h_{1}^{(1)}\right)^{4}<\infty$, имеют место равенства

$$
\begin{gathered}
\mathbf{E} H_{1}^{(1)}=a, \quad \mathbf{D} H_{1}^{(1)}=\sigma^{2}, \quad \mathbf{E}\left(H_{1}^{(1)}-a\right)^{3}=a_{3}, \\
\mathbf{E} H_{1}^{(2)}=-a, \quad \mathbf{D} H_{1}^{(2)}=\sigma^{2}, \quad \mathbf{E}\left(H_{1}^{(2)}+a\right)^{3}=-a_{3}, \\
\sigma^{2}=\mathbf{E}\left(h_{1}^{(1)}-a\right)^{2}, \quad \sigma^{2}<\infty, \quad a_{3}=\mathbf{E}\left(h_{1}^{(1)}-a\right)^{3}, \quad\left|a_{3}\right|<\infty, \quad a_{3}=\gamma_{3},
\end{gathered}
$$

где $\gamma_{3}-$ кумулянт третьего порядка случайной величины $h_{1}^{(1)}-a$.

Применимость локальной предельной теоремы ([6], гл. VII, §3 и §1, гл. VI, §1) с использованием асимптотических разложений для последовательности решетчатых случайных величин, распределения которых совпадают либо с распределением $h_{1}^{(1)}$, либо с распределением $-h_{1}^{(1)}$, непосредственно проверяется.

Тогда имеет место соотношение

$$
\begin{aligned}
P(t, n-t)=( & \left.\sigma n^{1 / 2}\right)^{-1}\left((2 \pi)^{-1 / 2} \exp \left\{-x_{t, n}^{2} / 1\right\}\right. \\
& +n^{-1 / 2}(2 \pi)^{-1 / 2} \exp \left\{-x_{t, n}^{2} / 2\right\} H_{3}\left(-x_{t, n}\right) \frac{\gamma_{3}}{6 \sigma^{2} a} x_{t, n} n^{-1 / 2} \\
& +n^{-1}(2 \pi)^{-1 / 2} \exp \left\{-x_{t, n}^{2} / 2\right\} \\
& \left.\times\left(H_{6}\left(-x_{t, n}\right) \frac{\gamma_{3}^{2}}{72 \sigma^{4} a^{2}} x_{t, n}^{2} n^{-1}+H_{4}\left(-x_{t, n}\right) \frac{\gamma_{4}}{24 \sigma^{4}}\right)+o\left(n^{-1}\right)\right),
\end{aligned}
$$

где

$$
x_{t, n}=(2 t-n)\left((\sigma / a) n^{1 / 2}\right)^{-1}=(t-2 n / 4)((\sigma / a) \sqrt{n / 4})^{-1}, \quad 1 \leqslant t \leqslant n-1,
$$

и $\gamma_{4}-$ кумулянт четвертого порядка случайной величины $\left(h_{1}^{(1)}-a\right)$ (он конечен в силу конечности $\left.\mathbf{E}\left(h_{1}^{(1)}\right)^{4}\right), H_{m}(x)$ - полином Чебышева-Эрмита степени $m$.

Положим

$$
\Delta_{n}=(\sigma / a \sqrt{(n / 4)})^{-1}
$$

Тогда из асимптотического представления вероятностей $P(t, n-t)$ вытекают следующие 3 Дискретная математика, т.22 №3 
соотношения для величин $Q_{n}(k, r)$ :

$$
\begin{aligned}
& Q_{n}(k, r)=\sum_{t=}^{r}(2 \pi)^{-1 / 2} \exp \left\{-x_{t, n}^{2} / 2\right\} \sigma^{-1} n^{-1 / 2} \\
&+ \sum_{t=k}^{r}(2 \pi)^{-1 / 2} \exp \left\{-x_{t, n}^{2} / 2\right\} H_{3}\left(-x_{t, n}\right) \frac{\gamma_{3}}{6 \sigma^{2} a} x_{t, n} \sigma^{-1} n^{-3 / 2} \\
&+\sum_{t=k}^{r}(2 \pi)^{-1 / 2} \exp \left\{-x_{t, n}^{2} / 2\right\} H_{6}\left(-x_{t, n}\right) \frac{\gamma_{3}^{2}}{72 \sigma^{4} a^{2}} x_{t, n}^{2} \sigma^{-1} n^{-5 / 2} \\
&+\sum_{t=k}^{r}(2 \pi)^{-1 / 2} \exp \left\{-x_{t, n}^{2} / 2\right\} H_{4}\left(-x_{t, n}\right) \frac{\gamma_{4}}{24 \sigma^{4}} \sigma^{-1} n^{-3 / 2}+o\left(|r-k| n^{-3 / 2}\right) \\
&=(2 a)^{-1} \sum_{t=k}^{r}(2 \pi)^{-1 / 2} \exp \left\{-1 / 2 x_{t, n}^{2}\right\} \Delta_{n} \\
&+\sum_{t=k}^{r}(2 \pi)^{-1 / 2} \exp \left\{-1 / 2 x_{t, n}^{2}\right\} H_{3}\left(-x_{t, n}\right) x_{t, n} \Delta_{n} \frac{\gamma_{3}}{12 \sigma^{2} a^{2}} n^{-1} \\
&+\sum_{t=k}^{r}(2 \pi)^{-1 / 2} \exp \left\{-x_{t, n}^{2} / 2\right\} H_{6}\left(-x_{t, n}\right) x_{t, n}^{2} \Delta_{n} \frac{\gamma_{3}^{2}}{144 \sigma^{4} a^{3}} n^{-2} \\
&+\sum_{t=k}^{r}(2 \pi)^{-1 / 2} \exp \left\{-1 / 2 x_{t, n}^{2}\right\} H_{4}\left(-x_{t, n}\right) \Delta_{n} \frac{\gamma_{4}}{48 \sigma^{4} a} n^{-1}+o\left(|r-k| n^{-3 / 2}\right) .
\end{aligned}
$$

Суммы

$$
\begin{aligned}
& \sum_{t=k}^{r}(2 \pi)^{-1 / 2} \exp \left\{-x_{t, n}^{2} / 2\right\} H_{3}\left(-x_{t, n}\right) x_{t, n} \Delta_{n}, \\
& \sum_{t=k}^{r}(2 \pi)^{-1 / 2} \exp \left\{-x_{t, n}^{2} / 2\right\} H_{6}\left(-x_{t, n}\right) x_{t, n}^{2} \Delta_{n}, \\
& \sum_{t=k}^{r}(2 \pi)^{-1 / 2} \exp \left\{-x_{t, n}^{2} / 2\right\} H_{4}\left(-x_{t, n}\right) \Delta_{n}
\end{aligned}
$$

суть части интегральных сумм с шагом разбиения $\Delta_{n}$ для абсолютно сходящихся интегралов (с ограниченными подынтегральными функциями)

$$
\begin{gathered}
(2 \pi)^{-1 / 2} \int_{-\infty}^{\infty} \exp \left\{-x^{2} / 2\right\} H_{3}(-x) x d x, \\
(2 \pi)^{-1 / 2} \int_{-\infty}^{\infty} \exp \left\{-x^{2} / 2\right\} H_{6}(-x) x^{2} d x, \\
(2 \pi)^{-1 / 2} \int_{-\infty}^{\infty} \exp \left\{-x^{2} / 2\right\} H_{4}(-x) d x
\end{gathered}
$$

соответственно. Следовательно, рассматриваемые суммы ограничены.

Сумма

$$
\sum_{t=k}^{r}(2 \pi)^{-1 / 2} \exp \left\{-x_{t, n}^{2} / 2\right\} \Delta_{n}
$$


есть интегральная сумма с шагом разбиения $\Delta_{n}$ для абсолютно сходящегося интеграла (с ограниченной подынтегральной функцией)

$$
(2 \pi)^{-1 / 2} \int_{x_{k, n}}^{x_{r, n}} \exp \left\{-x^{2} / 2\right\} d x
$$

Используя неравенства для оценки интегральных сумм (см. [7], с. 132), можно утверждать, что данный интеграл отличается от рассматриваемой интегральной суммы на величину порядка шага разбиения $\Delta_{n}$, то есть на величину $O\left(n^{-1 / 2}\right)$. Поэтому при $n \rightarrow \infty$ для величины $Q_{n}(k, r), k \geqslant r$, имеет место представление

$$
Q_{n}(k, r)=(2 a)^{-1}(2 \pi)^{-1 / 2} \int_{x_{k, n}}^{x_{r, n}} \exp \left\{-x^{2} / 2\right\} d x+O\left(n^{-1 / 2}\right)+o\left(|k-r| n^{-3 / 2}\right) .
$$

Далее, при $n \rightarrow \infty$

$$
\begin{aligned}
Q_{n}(1, n-1) & =(2 a)^{-1}(2 \pi)^{-1 / 2} \int_{x_{1, n}}^{x_{n-1, n}} \exp \left\{-x^{2} / 2\right\} d x+O\left(n^{-1 / 2}\right) \\
& =(2 a)^{-1}+O\left(n^{-1 / 2}\right),
\end{aligned}
$$

поскольку $x_{1, n}=-x_{n-1, n}, x_{n-1, n} \geqslant 0,\left|x_{n-1, n}\right|=O\left(n^{1 / 2}\right)$, и

$$
(2 \pi)^{-1 / 2} \int_{x_{1, n}}^{x_{n-1, n}} \exp \left\{-x^{2} / 2\right\} d x=1-\varepsilon_{n},
$$

где $\varepsilon_{n}>0$ и $\varepsilon_{n}=O\left(n^{-1 / 2}\right)$. Отсюда получаем, что при $N \rightarrow \infty$

$$
\sum_{n=2}^{N} Q_{n}(1, n-1)=(2 a)^{-1} N+O\left(N^{1 / 2}\right) \text {. }
$$

Далее, при $n \rightarrow \infty, n>N$,

$$
Q_{n}(1, N)=(2 a)^{-1}(2 \pi)^{-1 / 2} \int_{x_{1, n}}^{x_{N, n}} \exp \left\{-x^{2} / 2\right\} d x+O\left(n^{-1 / 2}\right)+o\left(N n^{-3 / 2}\right) .
$$

Согласно условию теоремы, при $N, M \rightarrow \infty$ величина $(N-M) /(N+M)^{1 / 2}$ стремится к конечному числу $\lambda$, откуда следует, что величина $M$ имеет порядок величины $N$. Тогда при $N \rightarrow \infty, N<n \leqslant M$, величина $x_{1, n}$ будет отрицательной и $x_{1, n}=O\left(N^{1 / 2}\right)$, а величина $x_{N, n}$ будет положительной и $x_{N, n}=O\left(N^{1 / 2}\right)$. Здесь

$$
(2 \pi)^{-1 / 2} \int_{x_{1, n}}^{x_{N, n}} \exp \left\{-x^{2} / 2\right\} d x=1+O\left(N^{-1 / 2}\right),
$$

поэтому отсюда получаем, что при $N, M \rightarrow \infty$

$$
\begin{aligned}
\sum_{n=N+1}^{M} Q_{n}(1, N) & =(2 a)^{-1}(M-N)+O\left(|M-N| N^{-1 / 2}\right)+o\left(|M-N| N \cdot N^{-3 / 2}\right) \\
& =(2 a)^{-1}(M-N)+O\left(|M-N| N^{-1 / 2}\right) .
\end{aligned}
$$


Далее, при $n \rightarrow \infty(M<n \leqslant M+N)$

$$
\begin{aligned}
Q_{n}(n-M, N)=(2 a)^{-1}(2 \pi)^{-1 / 2} \int_{x_{n-M, n}}^{x_{N, n}} \exp \left\{-x^{2} / 2\right\} d x \\
\quad+O\left(n^{-1 / 2}\right)+o\left(|N+M-n| n^{-3 / 2}\right) .
\end{aligned}
$$

Из условия теоремы, состоящего в том, что величина $(N-M) /(N+M)^{1 / 2}$ стремится при $N, M \rightarrow \infty$ к конечному числу $\lambda$, следует, что величина $N$ имеет порядок величины $M$. Тогда при $M \rightarrow \infty, M<n \leqslant M+N$, величина $x_{n-M, n}$ будет неположительной и иметь порядок $\left|(n / 2-M) n^{-1 / 2}\right|$, а величина $x_{N, n}$ будет иметь порядок $\left|(N-n / 2) n^{-1 / 2}\right|$. При $x \rightarrow \infty$

$$
(2 \pi)^{-1 / 2} \int_{-\infty}^{x} \exp \left\{-y^{2} / 2\right\} d y=1-\varepsilon(x),
$$

где $\varepsilon(x)>0$ и $\varepsilon=O\left(x^{-1}\right)$, поэтому при $N, M \rightarrow \infty$

$$
\begin{aligned}
& \sum_{n=M+1}^{M+N} Q_{n}(n-M, N)=(2 a)^{-1}(2 N-M)+O\left(\sum_{n=M+1}^{2 N-1}\left|n^{1 / 2}(N-n / 2)^{-1}\right|\right)+O(1) \\
&+O\left(\sum_{n=2 N+1}^{M+N}\left|n^{1 / 2}(N-n / 2)^{-1}\right|\right) \\
&+O\left(\sum_{n=M+1}^{M+N}\left|n^{1 / 2}(n / 2-M)^{-1}\right|\right)+O\left(\sum_{n=M+1}^{M+N} n^{-1 / 2}\right) \\
&+O\left(\sum_{n=M+1}^{M+N}(N+M-n) M^{-3 / 2}\right) \\
&=(2 a)^{-1} N+(2 a)^{-1}(N-M)+O\left((M+N)^{1 / 2} \ln (N+M)\right)+O\left((M+N)^{1 / 2}-M^{1 / 2}\right)+O\left(N^{2} M^{-3 / 2}\right) \\
&=(2 a)^{-1} N+O\left((M+N)^{1 / 2} \ln (N+M)\right) .
\end{aligned}
$$

Окончательно, учитывая все полученные оценки, получаем, что

$$
\begin{aligned}
\mathbf{E} L_{N, M}= & (2 a)^{-1} N+O\left(N^{1 / 2}\right)+(2)^{-1}(M-N)+O\left((N-M) N^{-1 / 2}\right) \\
& +(2 a)^{-1} N+O\left((M+N)^{1 / 2} \ln (N+M)\right) \\
= & (2 a)^{-1}(N+M)+O\left((M+N)^{1 / 2} \ln (N+M)\right) .
\end{aligned}
$$

Доказательство теоремы закончено.

В работе [5] исследовалась последовательность независимых одинаково распределенных случайных векторов $\left\{\left(\eta_{k}^{(1)}, \eta_{k}^{(2)}\right)\right\}_{k=1}^{\infty}$, где случайные величины $\eta_{k}^{(1)}, \eta_{k}^{(2)}$ принимают натуральные значения и суть последовательные промежутки между совпадениями $\tau_{1}$ и $\tau_{2}$, то есть, выполняются равенства

$$
\tau_{1} \sum_{j=1}^{k} \eta_{j}^{(1)}=\tau_{2} \sum_{j=1}^{k} \eta_{j}^{(2)}
$$


для любого $k \geqslant 1$ и $\tau_{1}\left(t_{1}\right) \neq \tau_{2}\left(t_{2}\right)$, если

$$
\sum_{j=1}^{k-1} \eta_{j}^{(i)}<t_{i}<\sum_{j=1}^{k} \eta_{j}^{(i)}, \quad \sum_{j=1}^{0} \eta_{j}^{(i)}=0, \quad i=1,2 .
$$

Имеют место равенства

$$
\mathbf{E} \eta_{k}^{(1)}=\mathbf{E} \eta_{k}^{(2)}=a
$$

(cм. [5]).

Верна следующая теорема.

Теорема 2. Пусть выполняются следующие условия:

(1) существует конечная ненулевая дисперсия $\sigma^{2}=\mathbf{D} h_{1}^{(1)}=\mathbf{D} h_{1}^{(2)}$,

(2) существует конечная ненулевая дисперсия $\Lambda^{2}=\mathbf{D} \eta_{1}^{(1)}=\mathbf{D} \eta_{1}^{(2)}$,

(3) $п р и ~ N, M \rightarrow \infty$ величина $(N-M) /(N+M)^{1 / 2}$ сходится к конечному числу $\lambda$.

Тогда для любого конечного $x$

$$
\mathbf{P}\left(\frac{L_{N, M}-(N+M) /(2 a)}{\sqrt{(N+M) \Lambda^{2} /\left(2 a^{3}\right)}} \geqslant x\right) \rightarrow \mathbf{P}\left(\xi_{1} \leqslant-x+\frac{\lambda}{\Lambda}(a / 2)^{1 / 2}, \xi_{2} \leqslant-x-\frac{\lambda}{\Lambda}(a / 2)^{1 / 2}\right),
$$

где случайный вектор $\left(\xi_{1}, \xi_{2}\right)$ распределен по нормальному закону с вектором средних значений $(0,0)$ и матрицей вторых моментов $\left(\begin{array}{ll}1 & \rho \\ \rho & 1\end{array}\right), \rho=1-\sigma^{2} /\left(\Lambda^{2} a\right)$.

Доказательство. Из определения случайных векторов $\left\{\left(\eta_{k}^{(1)}, \eta_{k}^{(2)}\right)\right\}_{k=1}^{\infty}$ следует справедливое при любом натуральном $n$ соотношение

$$
\mathbf{P}\left(L_{N, M} \geqslant n\right)=\mathbf{P}\left(\sum_{k=1}^{n} \eta_{k}^{(1)} \leqslant N, \sum_{k=1}^{n} \eta_{k}^{(2)} \leqslant M\right) .
$$

Пусть $x-$ некоторое число. Положим

$$
\hat{n}=\left[(N+M) /(2 a)+x \sqrt{(N+M) \Lambda^{2}\left(2 a^{3}\right)}\right],
$$

где $[y]-$ целая часть числа $y$; если $N, M \rightarrow \infty$, то $\hat{n} \rightarrow \infty$, и тогда

$$
\begin{aligned}
& \mathbf{P}\left(\frac{L_{N, M}-(N+M) /(2 a)}{\sqrt{(N+M) \Lambda^{2} /\left(2 a^{3}\right)}} \geqslant x\right) \\
& =\mathbf{P}\left(\sum_{k=1}^{\hat{n}} \frac{\left(\eta_{k}^{(1)}-a\right)}{\Lambda \hat{n}^{1 / 2}} \leqslant \frac{N-\hat{n} a}{\sqrt{\Lambda^{2} \hat{n}}}, \sum_{k=1}^{\hat{n}} \frac{\left(\eta_{k}^{(2)}-a\right)}{\Lambda \hat{n}^{1 / 2}} \leqslant \frac{M-\hat{n} a}{\sqrt{\Lambda^{2} \hat{n}}}\right) .
\end{aligned}
$$

Из условий теоремы следует, что

$$
\begin{gathered}
\frac{N-\hat{n} a}{\sqrt{\Lambda^{2} \hat{n}}}=\frac{(N-M) / 2-x \sqrt{\left(\Lambda^{2} /(2 a)\right)(N+M)}+O(1)}{\sqrt{\left(\Lambda^{2} /(2 a)\right)(N+M)\left(1+O\left(x(N+M)^{-1 / 2}\right)+O(1)\right.}} \rightarrow \frac{\lambda}{\Lambda}\left(\frac{a}{2}\right)^{1 / 2}-x, \\
\frac{M-\hat{n} a}{\sqrt{\Lambda^{2} \hat{n}}}=\frac{-(N-M) / 2-x \sqrt{\left(\Lambda^{2} /(2 a)\right)(N+M)}+O(1)}{\sqrt{\left(\Lambda^{2} /(2 a)\right)(N+M)\left(1+O\left(x(N+M)^{-1 / 2}\right)+O(1)\right.}} \rightarrow \frac{\lambda}{\Lambda}\left(\frac{a}{2}\right)^{1 / 2}-x, \\
\left.\mathbf{E}\left(\frac{\eta_{k}^{(1)}-a}{\Lambda}\right)\left(\frac{\eta_{k}^{(2)}-a}{\Lambda}\right)=\Lambda^{-2} \mathbf{E}\left(\eta_{k}^{(1)} \eta_{k}^{(2)}\right)-a^{2}\right) .
\end{gathered}
$$


В работе [5] получено соотношение

$$
\mathbf{E}\left(\eta_{k}^{(1)}-\eta_{k}^{(2)}\right)^{2}=2 \sigma^{2} / a .
$$

Отсюда следует, что

$$
\mathbf{E}\left(\eta_{k}^{(1)} \eta_{k}^{(2)}\right)=\Lambda^{2}+a^{2}-\sigma^{2} / a .
$$

Поэтому

$$
\mathbf{E}\left(\frac{\eta_{k}^{(1)}-a}{\Lambda}\right)\left(\frac{\eta_{k}^{(2)}-a}{\Lambda}\right)=\Lambda^{-2}\left(\Lambda^{2}+a^{2}-\sigma^{2} / a-a^{2}\right)=1-\frac{\sigma^{2}}{\Lambda^{2} a}
$$

Справедлива оценка

$$
\rho=\mathbf{E}\left(\frac{\eta_{k}^{(1)}-a}{\Lambda}\right)\left(\frac{\eta_{k}^{(2)}-a}{\Lambda}\right)=1-\frac{\sigma^{2}}{\Lambda^{2} a}<1
$$

При этом $\rho \neq-1$, поскольку иначе

$$
\mathbf{D}\left(\frac{\eta_{k}^{(1)}-a}{\Lambda}+\left(\frac{\eta_{k}^{(2)}-a}{\Lambda}\right)\right)=2+2 \rho=0
$$

и событие $\left\{\eta_{k}^{(1)}+\eta_{k}^{(2)}=2 a\right\}$ должно происходить на множестве единичной меры, что не так в силу определения случайных величин $\eta_{k}^{(1)}, \eta_{k}^{(2)}$, в частности,

$$
\mathbf{P}\left(\eta_{k}^{(1)}+\eta_{k}^{(2)}=2\right)=\mathbf{P}\left(\eta_{k}^{(1)}=1, \eta_{k}^{(2)}=1\right)=\mathbf{P}\left(h_{1}^{(1)}=h_{1}^{(2)}\right)>0 .
$$

Последовательность случайных двумерных векторов

$$
\left\{\frac{\eta_{k}^{(1)}-a}{\Lambda}, \frac{\eta_{k}^{(2)}-a}{\Lambda}\right\}_{k=1}^{\infty}
$$

есть последовательность независимых одинаково распределенных случайных векторов, у которых компоненты имеют нулевые математические ожидания, единичные дисперсии и коэффициент корреляции $\rho(\rho \neq 1,-1)$. Поэтому, как нетрудно убедиться, последовательность характеристических функций, соответствующая последовательности случайных двумерных векторов

$$
\left\{\sum_{k=1}^{n} \frac{\eta_{k}^{(1)}-a}{\Lambda n^{1 / 2}}, \sum_{k=1}^{n} \frac{\eta_{k}^{(2)}-a}{\Lambda n^{1 / 2}}\right\}_{n=1}^{\infty}
$$

при $n \rightarrow \infty$ сходится к функции

$$
\varphi\left(t_{1}, t_{2}\right)=\exp \left\{-\left(t_{1}^{2}+t_{2}^{2}+2 t_{1} t_{2} \rho / 2\right)\right\}
$$

которая является характеристической функцией случайного вектора $\left(\xi_{1}, \xi_{2}\right)$, распределенного по нормальному закону с вектором средних значений $(0,0)$ и матрицей вторых моментов $\left(\begin{array}{ll}1 & \rho \\ \rho & 1\end{array}\right)$.

Теорема доказана. 
Вопрос о конечности или бесконечности вторых моментов случайных величин $\eta_{1}^{(1)}$, $\eta_{1}^{(2)}$ в зависимости от существования у случайных величин $h_{1}^{(1)}, h_{1}^{(2)}$ моментов более высокого порядка, чем первый, остается в общем случае открытым.

Для частного случая случайных блужданий с приращениями 1 и 2 имеет место следующая теорема.

Теорема 3. Пусть $\mathbf{P}\left\{h_{1}^{(i)}=1\right\}=q, \mathbf{P}\left\{h_{1}^{(i)}=2\right\}=p, p+q=1,0<p<1, i=1,2$. тогда

$$
\begin{aligned}
& \mathbf{E} \eta_{1}^{(1)}=\mathbf{E} \eta_{1}^{(2)}=1+p \\
& \mathbf{D} \eta_{1}^{(1)}=\mathbf{D} \eta_{1}^{(2)}=p q+\frac{2 p^{3}}{q(1+p)} .
\end{aligned}
$$

Доказательство. Для данного случая

$$
\begin{aligned}
a & =\mathbf{E} h_{1}^{(1)}=\mathbf{E} h_{1}^{(2)}=q+2 p=1+p, \\
\sigma^{2} & =\mathbf{D} h_{1}^{(1)}=\mathbf{D} h_{1}^{(2)}=q+4 p-(1+p)^{2}=p q .
\end{aligned}
$$

Пусть $p(k, m)=\mathbf{P}\left(\eta_{1}^{(1)}=k, \eta_{1}^{(2)}=m\right)$ - вероятность того, что случайная величина $\eta_{1}^{(1)}$ равна $k$, а случайная величина $\eta_{1}^{(2)}$ равна $m$. Из определения следует, что

$$
0 \leqslant p(k, m) \leqslant 1, \quad \sum_{k=1}^{\infty} \sum_{m=1}^{\infty} p(k, m)=1 \text {. }
$$

Очевидно, что

$$
p(1,1)=p^{2}+q^{2}
$$

Если последовательность случайных величин $\left\{h_{1}^{(1)}, \ldots, h_{k}^{(1)}\right\}$ длины $k, k \geqslant 1$, приняла значения из всех $k$ двоек, то последовательность $\left\{\tau_{1}(1), \ldots, \tau_{1}(k)\right\}$ есть последовательность всех четных чисел от 2 до $2 k$. Если при этом в последовательности случайных величин $\left\{h_{1}^{(2)}, \ldots, h_{k}^{(2)}\right\}$ на первом месте стоит 1 , а на всех остальных 2, то последовательность $\left\{\tau_{2}(1), \ldots, \tau_{2}(k)\right\}$ есть последовательность всех нечетных чисел от 1 до $2 k-1$. Поэтому $\tau_{1}(t) \neq \tau_{2}(m)$ при $1 \leqslant t \leqslant k, 1 \leqslant m \leqslant k$. Если $h_{k+1}^{(2)}=1$, то $\tau_{1}(k)=\tau_{2}(k+1)$ впервые, то есть

$$
p(k, k+1) \geqslant p^{k} q p^{k-1} q=q^{2} p^{2 k-1}, k=1,2, \ldots
$$

Аналогично, для случая, когда последовательность $\left\{h_{1}^{(2)}, \ldots, h_{k}^{(2)}\right\}$ приняла значения из $k$ двоек, а в последовательности случайных величин $\left\{h_{1}^{(1)}, \ldots, h_{k}^{(1)}, h_{k+1}^{(1)}\right\}$ на первом месте стоит 1 , на последующих $k-1$ местах стоят 2 и $h_{k+1}^{(1)}=1$, получаем, что

$$
p(k+1, k) \geqslant q^{2} p^{2 k-1}, \quad k=1,2, \ldots
$$

Пусть последовательность случайных величин $\left\{h_{1}^{(1)}, \ldots, h_{k}^{(1)}, h_{k+1}^{(1)}\right\}$ длины $k+1$, $k \geqslant 1$, приняла значения из первых $k$ двоек, а величина $h_{k+1}^{(1)}$ приняла значение 1 . При этом последовательность случайных величин $\left\{h_{1}^{(2)}, \ldots, h_{k}^{(2)}, h_{k+1}^{(2)}\right\}$ длины $k+1, k \geqslant 1$, приняла 
значения из последних $k$ двоек, а $h_{1}^{(2)}$ приняла значение 1 . Тогда $\tau_{1}(k+1)=\tau_{2}(k+1)$ впервые. Аналогично, это произойдет, если в последовательности $\left\{h_{1}^{(2)}, \ldots, h_{k}^{(2)}, h_{k+1}^{(2)}\right\}$ на первых $k$ местах стоят 2 , на последнем месте 1 , а в последовательности $\left\{h_{1}^{(1)}, \ldots, h_{k}^{(1)}, h_{k+1}^{(1)}\right\}$ на первом месте стоит 1 , на остальных $k$ местах стоят 2. Поэтому

$$
p(k+1, k+1) \geqslant 2 p^{k} q p^{k} q=2 q^{2} p^{2 k}, \quad k=1,2, \ldots
$$

Найдем сумму $S$ правых частей полученных неравенств и равенства для $p(1,1)$ :

$$
\begin{aligned}
S & =\sum_{k=1}^{\infty} q^{2} p^{2 k-1}+\sum_{k=1}^{\infty} q^{2} p^{2 k-1}+\sum_{k=1}^{\infty} 2 q^{2} p^{2 k}+p^{2}+q^{2} \\
& =p^{2}+q^{2}+2 q^{2} p \sum_{k=1}^{\infty}\left(p^{2}\right)^{k-1}+2 q^{2} p^{2} \sum_{k=1}^{\infty}\left(p^{2}\right)^{k-1} \\
& =p^{2}+q^{2}+2 q^{2} \frac{p}{1-p^{2}}+\frac{2 q^{2} p^{2}}{1-p^{2}}=p^{2}+q^{2}+2 q^{2} p \frac{1+p}{q(1+p)} \\
& =p^{2}+q^{2}+2 p q=(p+q)^{2}=1 .
\end{aligned}
$$

Отсюда следует, что полученные неравенства, на самом деле, являются равенствами, и других типов реализаций последовательностей $\left\{h_{1}^{(1)}, \ldots, h_{n}^{(1)}\right\}$ и $\left\{h_{1}^{(2)}, \ldots, h_{m}^{(2)}\right\}$ при какихто $n, m, n, m \geqslant 1$, при которых состоялись бы события $\left\{\eta_{1}^{(1)}=k, \eta_{1}^{(2)}=l\right\}, 1 \leqslant k \leqslant n$, $1 \leqslant l \leqslant m)$, нет. Получим выражение для производящей функции $P(x, y)$ вероятностей $\{p(k, m)\},|x| \leqslant 1,|y| \leqslant 1$ :

$$
\begin{aligned}
P(x, y)= & \sum_{k=1}^{\infty} \sum_{m=1}^{\infty} x^{k} y^{m} p(k, m) \\
= & x y\left(p^{2}+q^{2}\right)+\sum_{k=1}^{\infty} x^{k} y^{k+1} q^{2} p^{2 k-1}+\sum_{k=1}^{\infty} x^{k+1} y^{k} q^{2} p^{2 k-1} \\
& \quad+\sum_{k=1}^{\infty} x^{k+1} y^{k+1} 2 q^{2} p^{2 k} \\
= & x y\left(p^{2}+q^{2}\right)+\sum_{k=1}^{\infty} q^{2} x^{k} y^{k}(x+y) p^{2 k-1}+2 q^{2} p^{2} x^{2} y^{2} \sum_{k=1}^{\infty}\left(x y p^{2}\right)^{k-1} \\
= & x y\left(p^{2}+q^{2}\right)+(x+y) q^{2} p x y \sum_{k=1}^{\infty}\left(x y p^{2}\right)^{k-1}+2 q^{2} p^{2} x^{2} y^{2} \frac{1}{1-x y p^{2}} \\
= & x y\left(p^{2}+q^{2}\right)+q^{2} p(x+y) x y \frac{1}{1-x y p^{2}}+2 q^{2} p^{2} x^{2} y^{2} \frac{1}{1-x y p^{2}} \\
= & x y\left(p^{2}+q^{2}+(x+y+2 p x y) \frac{q^{2} p}{1-x y p^{2}}\right) .
\end{aligned}
$$

Теперь можно получить выражение для производящей функции $P_{1}(x)$ случайной ве- 
личины $\eta_{1}^{(1)}$ :

$$
\begin{aligned}
P_{1}(x) & =P(x, 1)=x\left(p^{2}+q^{2}+(x+y+2 p x) \frac{q^{2} p}{1-x p^{2}}\right) \\
& =x\left(p^{2}+q^{2}\right)+x \frac{q^{2} p}{1-x p^{2}}\left(1-x p^{2}+x+x p^{2}+2 x p\right) \\
& =x\left(p^{2}+q^{2}+q^{2} p\right)+x^{2} \frac{q^{2} p(1+p)^{2}}{1-x p^{2}} .
\end{aligned}
$$

Аналогично получается выражение для производящей функции $P_{2}(x)$ случайной величины $\eta_{1}^{(2)}$ :

$$
P_{2}(y)=P(1, y)=y\left(p^{2}+q^{2}+q^{2} p\right)+y^{2} \frac{q^{2} p(1+p)^{2}}{1-y p^{2}}
$$

Далее получаем, что

$$
\begin{aligned}
P_{1}^{\prime}(x) & =p^{2}+q^{2}+q^{2} p+q^{2} p(1+p)^{2} \frac{2 x}{1-x p^{2}}+q^{2} p(1+p)^{2} \frac{p^{2} x^{2}}{\left(1-x p^{2}\right)^{2}} \\
& =p^{2}+q^{2}+q^{2} p+q^{2} p(1+p)^{2} \frac{2 x-p^{2} x^{2}}{\left(1-x p^{2}\right)^{2}} \\
P_{1}^{\prime \prime}(x) & =q^{2} p(1+p)^{2}\left(\frac{2 p^{2}}{\left(1-x p^{2}\right)^{3}}\left(2 x-p^{2} x^{2}\right)+\frac{1}{\left(1-x p^{2}\right)^{2}}\left(2-2 p^{2} x\right)\right) .
\end{aligned}
$$

Отсюда получаются выражения для математических ожиданий и дисперсий случайных величин $\eta_{1}^{(1)}, \eta_{1}^{(2)}$ :

$$
\begin{aligned}
\mathbf{E} \eta_{1}^{(1)} & =\mathbf{E} \eta_{1}^{(2)}=P_{1}^{\prime}(1)=p^{2}+q^{2}+q^{2} p+q^{2} p(1+p)^{2} \frac{2-p^{2}}{\left(1-p^{2}\right)^{2}} \\
& =p^{2}+q^{2}+q^{2} p+p\left(2-p^{2}\right)=p^{2}+q^{2}+q^{2} p+p+p\left(1-p^{2}\right) \\
& =p^{2}+q^{2}+q^{2} p+p+p q(1+p)=p^{2}+q^{2}+q^{2} p+p+p^{2} q+p q \\
& =p^{2}+q^{2}+p+q p(p+q)+p q=p^{2}+q^{2}+2 q p+p \\
& =(p+q)^{2}+p=1+p ; \\
\mathbf{E} \eta_{1}^{(1)}\left(\eta_{1}^{(1)}-1\right) & =\mathbf{E} \eta_{1}^{(2)}\left(\eta_{1}^{(2)}-1\right)=P_{1}^{\prime \prime}(1) \\
& =q^{2} p(1+p)^{2}\left(\frac{2 p^{2}}{\left(1-p^{2}\right)^{3}}\left(2-p^{2}\right)+\frac{1}{\left(1-p^{2}\right)^{2}}\left(2-2 p^{2}\right)\right) \\
& =q^{2} p(1+p)^{2}\left(\frac{2 p^{2}}{q^{3}(1+p)^{3}}\left(1+1-p^{2}\right) \frac{2}{q(1+p)}\right) \\
& =p\left(\frac{2 p^{2}(1+q(1+p))}{q(1+p)}+2 q(1+p)\right) \\
& =p\left(\frac{2 p^{2}}{q(1+p)}+2 p^{2}+2 q(1+p)\right) \\
& =\frac{2 p^{3}}{q(1+p)}+2 p^{3}+2 p\left(1-p^{2}\right) \frac{2 p^{3}}{q(1+p)}+2 p,
\end{aligned}
$$


наконец,

$$
\begin{aligned}
\mathbf{D} \eta_{1}^{(1)} & =\mathbf{D} \eta_{1}^{(2)}=\mathbf{E} \eta_{1}^{(1)}\left(\eta_{1}^{(1)}-1\right)+\mathbf{E} \eta_{1}^{(1)}-\left(\mathbf{E} \eta_{1}^{(1)}\right)^{2} \\
& =\frac{2 p^{3}}{q(1+p)}+2 p+(1+p)-(1+p)^{2} \\
& =\frac{2 p^{3}}{q(1+p)}+p-p^{2}=p q+\frac{2 p^{3}}{q(1+p)} .
\end{aligned}
$$

На этом доказательство теоремы закончено.

Из теоремы 3 следует, что для случайных блужданий с приращениями 1 и 2 выполняются первые два условия теоремы 2, а при выполнении третьего условия, накладывающего ограничения на поведение величин $N$ и $M$, будет следовать утверждение теоремы 2 для данного частного случая.

\section{Список литературы}

1. Chvatal V., Sankov D., Longest common subsequences of two random sequences. J. Appl. Probab. (1975) 12, 306-315.

2. Михайлов В. Г., Меженная Н. М., Оценки для вероятностей плотного вложения одной дискретной последовательности в другую. Дискретная математика (2005) 17, №3, 19-27.

3. Меженная Н. М., Предельные теоремы для числа плотных серий в случайной последовательности. Дискретная математика (2009) 21, №1, 105-116.

4. Golic J. D., Constrained embedding probability for two binary strings. SIAM J. Discrete Math. (1996) 9, 360-364.

5. Кравченко И. А., О последовательности общих точек однородных случайных блужданий с положительными приращениями и их использование для оценки параметров одного способа скрытой передачи информации. Обозрение прикладной и промышленной математики (2007) 14, 598-609.

6. Петров В. В., Суммы независимых случайныхх величин. Наука, Москва, 1972.

7. Сачков В. Н., Введение в комбинаторные методы дискретной математики. Наука, Москва, 1982.

Статья поступила 20.02.2009. 